Table 2. Association of subject characteristics with perceived importance by race

\begin{tabular}{|c|c|c|c|}
\hline Variable & Non-Hispanic Whites & Non-Hispanic Blacks & Hispanics \\
\hline Medication beliefs ${ }^{1}$ & $-0.55(<0.01)$ & $-0.24(\mathbf{0 . 0 1})$ & $-0.08(0.46)$ \\
\hline Hopeful $^{1}$ & $0.54(<0.01)$ & $0.16(0.09)$ & $0.37(<0.01)$ \\
\hline Worried $^{1}$ & $-0.27(\mathbf{0 . 0 1 )}$ & $-0.02(0.87)$ & $0.05(0.66)$ \\
\hline Trust $^{1}$ & $0.22(\mathbf{0 . 0 2})$ & $0.33(\mathbf{0 . 0 1 )}$ & $0.17(0.11)$ \\
\hline Poor self-reported health ${ }^{2}$ & $1.66(0.10)$ & $1.19(0.25)$ & $-1.4(0.16)$ \\
\hline Some college education ${ }^{2}$ & $-1.16(0.25)$ & $1.9(0.06)$ & $0.65(0.52)$ \\
\hline Income $<12,000 /$ year $^{2}$ & $0.38(0.71)$ & $1.65(0.11)$ & $0.06(0.96)$ \\
\hline Difficulty paying for meds ${ }^{2}$ & $0.00(0.99)$ & $-2.05(0.04)$ & $-0.42(0.68)$ \\
\hline
\end{tabular}

${ }^{1}$ Correlation coefficient ( $p$ value); ${ }^{2}$ T-test ( $p$ value).

subjects had lower levels of hope compared to Non-Hispanic White subjects (difference between means $<0.05$ ). Associations between subject characteristics and perceived importance of taking the medication are presented in Table 2. In a multilinear regression model (including education, difficulty paying for medications, medication beliefs, trust, hope and worry), hope was associated with perceived importance of taking the medication in all three ethnic groups. Additional findings differed by race, with medication beliefs in Non-Hispanic White subjects; difficulty paying for medications in Non-Hispanic Black subjects, and worry in Hispanic subjects being associated with perceived importance of taking the medication.

Conclusions: Our findings confirm the important influence of emotion on decision making, and suggest that while hope is universally associated with perceived importance of taking a medication, other factors differed, highlighting differences in the decision making process across ethnic groups.

Disclosure of Interest: None declared

DOI: 10.1136/annrheumdis-2017-eular.2369

\section{AB1106 VERBAL PATIENT EDUCATION ON VACCINATION IN ADULTS WITH AUTOIMMUNE INFLAMMATORY RHEUMATIC DISEASES: IS IT ENOUGH TO IMPROVE VACCINATION RATES?}

R. Magro, M. Rogers, F. Camilleri. Rheumatology, Mater Dei Hospital, Msida, Malta

Background: Patients suffering from autoimmune inflammatory rheumatic diseases (AIIRD) are at increased risk of infections, due to the underlying condition and its treatment. A study on vaccination in AIIRD carried out at Mater Dei Hospital, Malta in 2015, showed that $37.9 \%$ of the patients received the influenza vaccine in the previous year and $8.3 \%$ had received the pneumococcal vaccine ever. Only $38.3 \%$ knew that they had an increased risk of infection because of their condition.

Objectives: The aim of the current study was to establish whether verbal education on vaccination, influenced patients' vaccination rates in the following year. A further aim was to determine whether the patients had a better understanding of their increased infection risk following verbal education.

Methods: The initial study carried out in 2015 consisted of a short face-to-face interview with 60 patients who suffered from autoimmune inflammatory rheumatic diseases. Following the interview, verbal information was given to the patients on their increased risk of infection, and the importance of vaccination, in particular the influenza and pneumococcal vaccine. After 1 year, the patients were contacted by means of a telephone call. They were interviewed with regards to their vaccination history and knowledge of their infection risk. Of the 60 patients, 2 had passed away. Therefore 58 patients were included in the study.

Results: The study included patients with a variety of AIIRD including rheumatoid arthrtitis, ankylosing spondylitis and psoriatic arthritis. $60.3 \%$ were females and the mean age was 63.1 years (range 25 to 82 years). Influenza vaccination rates in the previous year improved from $37.9 \%$ in 2015 to $41.4 \%$ in 2016 ( $p=0.704)$. Pneumococcal vaccination rates improved from $6.9 \%$ to $17.2 \%(p=0.086)$. On questioning the patients regarding their knowledge of increased infection risk, there was no improvement following the verbal education. In fact $37.9 \%$ of patients questioned in 2015 , and $34.5 \%$ in 2016 knew that they were at increased risk of infection because of their condition. 33\% of patients studied in 2016 could recall that they had been advised to take vaccination because of their underlying condition or treatment. This improved from $7 \%$ in $2015(p<0.005)$. The proportion of patients who could recall the vaccination advice given was significantly higher in those below 61 years $(p<0.005)$. However, there were no significant gender difference $(p=0.147)$.

Conclusions: Verbal education resulted in more patients receiving the pneumococcal vaccination (although not statistically significant because of the small number involved). It also resulted in a significant increase in the number of patients who were able to recall that they were adviced to take vaccination because of their condition. However, only $33 \%$ could recall this after a year. Verbal education did not effect the number of patients who took the influenza vaccine and the patients' knowledge of their increased infection risk.

This study highlights that verbal education helps to improve vaccination rates in autoimmune inflammatory rheumatic diseases. However, the advice given tends to be forgotten by the majority of patients after one year. Thus repeated verbal education is necessary, particularly in elderly patients. Moreover other methods of education, such as written information in the form of leaflets, may be useful.

Disclosure of Interest: None declared

DOI: 10.1136/annrheumdis-2017-eular.1962

\section{AB1107 ASSESSMENT OF AGREEMENT BETWEEN SELF-REPORT INFLAMMATORY ARTHRITIS SYMPTOMS AND CORRESPONDING GP DIAGNOSIS IN PATIENTS WITH PMR}

S.L. Hider ${ }^{1,2}$, M. Blagojevic-Bucknall ${ }^{2}$, S. Muller ${ }^{2}$, K. Clarkson ${ }^{2}$, C. Mallen ${ }^{2}$. ${ }^{1}$ Haywood Academic Rheumatology Centre, Haywood Hospital, Staffordshire; ${ }^{2}$ Arthritis Research UK Primary Care Centre, Keele University, Keele, United Kingdom

Background: Polymyalgia rheumatica (PMR) is the commonest inflammatory condition among those aged 50 years and over, with a lifetime risk of $2.4 \%$ in females and $1.7 \%$ in males. Seronegative rheumatoid arthritis is an important differential diagnosis. It is thought a proportion of PMR patients develop an inflammatory arthritis, although prevalence estimates vary.

Objectives: To determine the proportion of PMR patients (1) reporting symptoms of possible inflammatory arthritis using a validated self-report inflammatory arthritis algorithm; and (2) who subsequently receive a diagnosis of inflammatory arthritis in their primary care medical records.

Methods: A cross-sectional questionnaire study was developed to investigate impact of PMR in primary care patients. All eligible adults aged $\geq 50$ years, registered with one of 150 participating practices with a first ever Read coded diagnosis of PMR in the last 3 years were mailed a questionnaire including items about socio-demographics, PMR characteristics, joint problems and comorbid conditions. Self-reported inflammatory arthritis (IA) was defined using a validated scoring algorithm (1) with positive responses coded as 1 and negative responses as -1 ; a score $>0$ is regarded as predictive of IA. IA score was calculated for those patients that had complete data on all required variables. A list of Read Codes relating to inflammatory arthritis, compiled by a rheumatologist and a GP, were searched for in primary care medical records of consenting respondents. Period of interest was from date of first ever PMR diagnosis in the medical records until survey mail-out.

Results: 704 eligible patients were identified and sent a questionnaire, with 550 $(78 \%)$ responding. Responders and non-responders did not differ significantly by age or gender. Medical records could be obtained for 385 patients, of whom 310 completed the questionnaire fully and were included in the analysis. IA score ranged from -8.4 to 2.5 , with $21(7 \%)$ patients being classed as having a positive IA score. 8 out of 310 patients had at least one inflammatory arthritis Read code recorded from date of first diagnosis of PMR in their medical record, although only 2 of these also fulfilled the self-report IA criteria.

Conclusions: In this PMR cohort the proportion subsequently diagnosed with an inflammatory arthritis was low $(2.5 \%)$ although more patients reported inflammatory symptoms. The agreement between self-reported inflammatory symptoms and Read Code diagnosed inflammatory arthritis was low.

References:

[1] Tavares R, Wells GA, Bykerk VP et al. A a parallel group cohort to determine the measurement properties of an early inflammatory arthritis detection tool. Rheumatology 2013;52:2077-2085.

Acknowledgements: This study represents independent research funded by the National Institute for Health Research (NIHR). CDM is funded by the National Institute for Health Research (NIHR) Collaborations for Leadership in Applied Health Research and Care West Midlands, the NIHR School for Primary Care Research and a NIHR Research Professorship in General Practice (NIHR-RP2014-04-026). The views expressed are those of the authors and not necessarily those of the NHS, NIHR or the Department of Health.

Disclosure of Interest: None declared

DOI: 10.1136/annrheumdis-2017-eular.5271

\section{AB1108 HANDLING OF DE NOVO ARTHRITIS IN ADULTS BY GENERAL PRACTITIONERS: A SURVEY IN A FRENCH COHORT}

S. Lecauchois ${ }^{1}$, S. Gentile ${ }^{2}$, P. Champsaur ${ }^{3}$, J.-P. Mattei ${ }^{4}$, L. Dillinger ${ }^{5}$, V. Goeb ${ }^{6}$ S. Guis ${ }^{4,7}$. ${ }^{1}$ Aix Marseille Université, AP-HM, Marseille Cedex 9, France; ${ }^{2}$ Public Health; ${ }^{3}$ Radiology; ${ }^{4}$ Rheumatology, Aix Marseille Université, AP-HM, Marseille Cedex 9; 5 General Practitioner, Aix-en-Provence;

${ }^{6}$ Rheumatology, CHU Amiens, Université de Picardie Jules Verne, Amiens; ${ }^{7} \mathrm{Crmbm}$-Cemerem UMR 7339, CNRS, Aix Marseille Université, Marseille, France

Background: A guide of good clinical practice regarding de novo arthritis in adults has been edited.

Objectives: The aim of this work is to identify whether the diagnostic and therapeutic tools proposed by general practitioners (GPs) to adults with a de novo arthritis is in line with the corresponding recommendations.

Methods: A questionnaire regarding the characteristics and the handling of de novo arthritis in adults has been submitted to 300 GPs chosen randomly from a French cohort database. The study period was from February 2015 to February 2016. Fifty seven GPs participated in the study (19\%).

Results: No particular specificity was identified in the GP group. The age of patients ranged between 40 and 75 yo. The prevalence of arthritis was less than $5 \%$ among the GPs patients. The relative number of each category of arthritis was rheumatoid arthritis (29\%), spondyloarthritis (14\%), PPR $(8 \%)$, other kinds of microcrystalline arthritis: chondrocalcinosis, (38.4\%), gout (11,6\%).

Joints with mechanic degenerative effusion related to osteo-arthritis might be misdiagnosed as an inflammatory arthritis.

The knee was the most frequently involved joint in arthritis. The initial paraclinical 